\title{
Surgical and Peri-Operative Considerations for Brain Metastases
}

\author{
Saksham Gupta ${ }^{1 *}$, Hassan Dawood ${ }^{1}$, Alexandra Giantini Larsen ${ }^{2}$, Luis Fandino ${ }^{3}$, \\ Erik H. Knelson ${ }^{4}$, Timothy R. Smith ${ }^{1}$, Eudocia Q. Lee ${ }^{5}$, Ayal Aizer ${ }^{6}$, Ian F. Dunn ${ }^{7}$ \\ and Wenya Linda Bi ${ }^{1}$ \\ ${ }^{1}$ Center for Skull Base and Pituitary Surgery, Department of Neurosurgery, Brigham and Women's Hospital, Harvard \\ Medical School, Boston, MA, United States, ${ }^{2}$ Department of Neurosurgery, Weill Cornell Medical College, New York City, \\ NY, United States, ${ }^{3}$ Department of Orthopedic Surgery, University of Utah Health Science Center, Salt Lake City, UT, \\ United States, ${ }^{4}$ Department of Medical Oncology, Dana Farber Cancer Institute, Harvard Medical School, Boston, MA, \\ United States, ${ }^{5}$ Center for Neuro-Oncology, Dana Farber Cancer Institute, Harvard Medical School, Boston, MA, United States, \\ ${ }^{6}$ Department of Radiation Oncology, Brigham and Women's Hospital, Harvard Medical School, Boston, MA, United States, \\ ${ }^{7}$ Department of Neurosurgery, Oklahoma University Health Sciences Center, Oklahoma City, OK, United States
}

OPEN ACCESS

Edited by:

Seunggu Jude Han, Natividad Medical Center, United States

Reviewed by:

Stephen Bowden, Oregon Health and Science University, United States

Domenique Müller, VU University Medical Center, Netherlands

*Correspondence: Saksham Gupta sgupta@bwh.harvard.edu

Specialty section: This article was submitted to Neuro-Oncology and Neurosurgical Oncology, a section of the journa Frontiers in Oncology

Received: 01 February 2021 Accepted: 16 April 2021

Published: 05 May 2021

Citation:

Gupta S, Dawood H, Giantini Larsen A, Fandino L, Knelson EH, Smith TR, Lee EQ, Aizer A, Dunn IF and Bi WL (2021)

Surgical and Peri-Operative Considerations for Brain Metastases.

Front. Oncol. 11:662943. doi: 10.3389/fonc.2021.662943
Introduction: Brain metastases are the most common brain tumors in adults, whose management remains nuanced. Improved understanding of risk factors for surgical complications and mortality may guide treatment decisions.

Methods: A nationwide, multicenter analysis was conducted with a retrospective cohort. Adult patients in the 2012-2015 American College of Surgeons National Surgical Quality Improvement Project (ACS NSQIP) databases who received a craniotomy for resection of brain metastasis were included.

Results: 3500 cases were analyzed, of which 17\% were considered frail and 24\% were infratentorial. The most common 30-day medical complications were venous thromboembolism (3\%, median time-to-event [TTE] 4.5 days), pneumonia (4\%, median TTE 6 days), and urinary tract infections (2\%, median TTE 5 days). Reoperation and unplanned readmission occurred in $5 \%$ and $12 \%$ of patients, respectively. Infratentorial approach and frailty were associated with reoperation before discharge (OR 2.0 for both; $p=0.01$ and $p=0.03$ respectively), but not after discharge. Infratentorial approaches conferred heightened risk for readmission for hydrocephalus (OR 5.1, $\mathrm{p}=0.02$ ) and reoperation for cerebrospinal fluid diversion (OR 7.1, p<0.001).Overall 30-day mortality was $4 \%$, with nearly three-quarters occurring after discharge. Pre-frailty and frailty were associated with increased odds for post-discharge mortality (OR 1.7 and 2.7, p<0.05), but not pre-discharge mortality. We developed a model to identify pre-/peri-operative variables associated with death, including frailty, thrombocytopenia, and high American Society of Anesthesiologists score (AUROC 0.75).

Conclusions: Optimization of metrics contributing to patient frailty and heightened surveillance in patients with infratentorial metastases may be considered in the perioperative period.

Keywords: brain metastasis, surgical risk, readmission, reoperation, complications, mortality 


\section{INTRODUCTION}

Solid tumor brain metastasis is associated with high morbidity and mortality. The overall annual incidence of brain metastasis amongst cancer patients ranges from $2-10 \%$ and varies greatly by primary cancer origin, tumor stage, and demographic factors including race and age (1-3). The most common sites of origin for brain metastasis are lung (39-56\%), breast (13-30\%), and melanoma $(6-11 \%)(1,4)$. The incidence of brain metastasis has increased over the past 20 years in parallel with improved metastasis detection and treatment of the primary cancer; as patients live longer, their risk of brain metastasis increases $(4,5)$. Among patients with brain metastases, median survival ranges from 3 months for pancreatic cancer, to 6-9 months for melanoma, to 6-13 months or longer for lung and breast cancer (6-9).

Standard management for brain metastases involves, in general, radiation therapy with or without surgical resection. Surgical resection of brain metastases is typically indicated for isolated or oligo-metastatic disease, symptomatic or large tumors causing mass effect, and radiation-resistant tumors $(10,11)$. Resecting a metastasis may reduce symptoms, prevent neurological damage, and increase survival in select cases (1215). However, surgery exposes a patient to the risks of an operation, hospitalization, as well as the recovery period. Especially for patients with finite life expectancy from their malignancy, the expected benefits should outweigh the potential risks to optimize quality of life.

We sought to investigate factors that contribute to adverse events following resection of brain metastases in order to inform selection of patients for surgery. We focused on tumor location and frailty in this analysis. Infratentorial tumors can present higher surgical risk due to their proximity to the brainstem and vital neurovascular structures. Frailty quantifies a patient's chronic disease burden and has been independently associated with poorer health outcomes in nonsurgical patients and adverse postoperative outcomes in other surgical fields (16-20).

\section{MATERIALS AND METHODS}

\section{Data Source}

The American College of Surgeons National Surgical Quality Improvement Project (NSQIP) 2012-2015 databases were obtained for this analysis. These databases contain validated, multi-institutional data collected in a standardized method across institutions by trained coders (21). The NSQIP databases contain cases from private and academic centers. In 2012, 374 hospitals provided data, which rose to 603 by 2015 .

\section{Inclusion and Exclusion Criteria}

The NSQIP database was filtered by relevant criteria. Inclusion required a billing code for brain metastasis (ICD9 198.3 or ICD10 C79.31), and a neurosurgical CPT code of 61510 , $61518,61520,61521,61526$, or 61530. Patients were included if they were at least 18 years old, had a neurosurgeon as the primary attending surgeon for their case, and received a procedure under general anesthesia. These criteria yielded 3567 cases. Of these, 67 were excluded for operative time under 30 minutes or post-operative length of stay of 0 days since these were assumed to be part of an aborted or miscoded procedure.

\section{Patient Stratification}

Pertinent preoperative and perioperative co-variates were obtained for each case. Sex, age, body mass index (BMI), case urgency, pre-operative co-morbidities, American Society of Anesthesiologist (ASA) class, and pre-operative laboratory abnormalities were included. Tumor location was extrapolated based on the associated current procedural terminology (CPT) code, with infratentorial (CPT 61518, 61520, 61521, 61526, or 61530) and supratentorial (CPT 61510) strata. These codes were initially derived to stratify procedures for billing purposes. Operative time was binned into quartiles.

The modified frailty index (mFI) with 5 co-variates (mFI-5) was calculated for each case as an estimate of patients' chronic disease burden with one point assigned for congestive heart failure (CHF), chronic obstructive pulmonary disease (COPD), diabetes mellitus (DM), hypertension (HTN), and nonindependent functional status. This scale is a modification of the validated $\mathrm{mFI}-11$, with strong concordance with the mFI-11 in prior nationwide analyses $(16,22)$. A mFi-5 score of 0 was considered non-frail, 1 as pre-frail, and $2+$ as frail.

NSQIP databases encode postoperative outcomes up to 30 days, including surgical site infections (SSIs), wound dehiscence, pneumonia, reintubation, deep vein thrombosis (DVT)/ pulmonary embolism (PE), urinary tract infections (UTI), cerebrovascular accidents (CVAs), cardiac arrests, myocardial infarction (MI), transfusions, sepsis, extended length of stay defined as over 1 week, reoperation, unplanned readmission, and death. Outcomes of interest were further stratified by whether they occurred during the index hospitalization or after initial discharge. Analyses of post-discharge complications excluded patients who died during the index hospitalization.

Complications encoded on the day of discharge were considered post-discharge as they would likely have prolonged hospitalizations had they occurred prior to a discharge. In contrast, death that was encoded on the day of discharge was considered pre-discharge given that a critical patient at risk of death would likely not have been discharged that same day.

\section{Statistical Analysis}

The R Package for Statistical Computing version 3.5.1 was utilized for all analyses. Cases with missing co-variate data were binned into "missing" categories and treated as a separate independent variable during all analyses. The impact of frailty and tumor location on all postoperative outcomes was calculated as odds ratios (OR) through multivariate regression with adjustment for sex, age, BMI, ASA class, transfer from outside hospital, tumor location, smoking, steroid usage, case urgency, pre-operative lab abnormalities, operative time, use of operative microscope and stereotactic navigation. A two-sided probability value ( $p$ ) of under 0.05 was utilized as the threshold for statistical significance. 
A predictive model for 30-day death was also developed. A univariate screen of all co-variates associated with death, excluding any co-variates with fewer than $0.1 \%$ of any level associated with a death, was conducted using a $\chi^{2}$ statistical significance cut-off of $\mathrm{p}<0.10$. The screened co-variates were included in a multivariate logistic regression with backwards stepwise variable elimination. The Hosmer-Lemeshow test and area under the receiver-operator curve (AUROC) concordance statistic was utilized to assess calibration and discrimination, respectively. The pre-determined value for an acceptable calibration for the model was $\mathrm{p} \geq 0.05$.

\section{RESULTS}

\section{Case Characteristics}

Within the study population, 3500 patients with brain metastases were included, with $55 \%$ being female (Table 1). Median age of this cohort was 61 years (Interquartile range [IQR] 54-69). Infratentorial lesions represented $24 \%$ of cases. Patients were

TABLE 1 | Clinical Characteristics.

\begin{tabular}{|c|c|c|}
\hline \multicolumn{3}{|l|}{ Total cases $=3500$} \\
\hline Sex & $\mathrm{N}$ & $\%$ \\
\hline Female & 1936 & 55.3 \\
\hline Age & $\mathrm{N}$ & $\%$ \\
\hline $18-50$ & 636 & 18.2 \\
\hline $50-65$ & 1619 & 46.3 \\
\hline $65-75$ & 949 & 27.1 \\
\hline $75+$ & 296 & 8.5 \\
\hline BMl & $\mathrm{N}$ & $\%$ \\
\hline Normal & 2365 & 67.6 \\
\hline Class I Obese & 611 & 17.5 \\
\hline Class II Obese & 382 & 10.9 \\
\hline Unknown & 142 & 4.1 \\
\hline Past Medical History & $\mathrm{N}$ & $\%$ \\
\hline $\mathrm{CHF}$ & 17 & 0.5 \\
\hline COPD & 432 & 12.3 \\
\hline DM & 413 & 11.8 \\
\hline HTN Meds & 1455 & 41.6 \\
\hline Smoker & 1042 & 29.8 \\
\hline Steroid Use & 860 & 24.6 \\
\hline Modified Frailty Index & $N$ & $\%$ \\
\hline $0=$ Non-Frail & 1672 & 47.8 \\
\hline $1=$ Pre-Frail & 1245 & 35.6 \\
\hline $2+=$ Frail & 583 & 16.7 \\
\hline ASA Class & $\mathrm{N}$ & $\%$ \\
\hline $1-2$ & 394 & 11.3 \\
\hline 3 & 2323 & 66.4 \\
\hline $4-5$ & 764 & 21.8 \\
\hline Unknown & 19 & 0.5 \\
\hline Location of Tumor & $\mathrm{N}$ & $\%$ \\
\hline Supratentorial & 2674 & 76.4 \\
\hline Infratentorial & 826 & 23.6 \\
\hline Location of Arrival & $\mathrm{N}$ & $\%$ \\
\hline Direct Admit & 2722 & 77.8 \\
\hline Transfer & 775 & 22.1 \\
\hline Unknown & 3 & 0.1 \\
\hline
\end{tabular}

ASA, American Society of Anesthesiologists; BMI, body mass index; CHF, congestive heart failure; COPD, chronic obstructive pulmonary disease; DM, Diabetes mellitus; HTN, hypertension; N, Number. considered non-frail ( $\mathrm{n}=1672,48 \%)$, pre-frail $(\mathrm{n}=1245,36 \%)$, or frail $(n=583,17 \%)$ as quantified by the mFI-5 index. Most patients were admitted to the same hospital where they presented $(n=2722,78 \%)$, and $50 \%$ of cases were electively scheduled. Median operative length was 139 minutes (IQR 98-193).

\section{Postoperative Course}

We first examined post-operative medical complications and interrogated their temporal patterns in an effort to identify risk factors for poor or unexpected outcomes. Median length of stay following operation was 3 days (IQR 2-5); 14\% of patients were hospitalized for longer than 7 days postoperatively. The most frequent postoperative medical complications overall were venous thromboembolism (VTE; including DVTs and PEs), pneumonia, UTI, and sepsis (Table 2). Stratifying complications by whether they occurred during index hospitalization or after initial discharge revealed temporal trends for specific complications (Table 2). Cardiac events (myocardial infarctions and cardiac arrests) and CVAs tended to occur within 0-2 days postoperatively and prior to discharge (Figure 1A). In contrast, the median time to occurrence for inhospital DVTs, infectious complications, and reoperations tended to be 4-6 days. Complications that arose after discharge occurred with a wide temporal spread (Figure 1A). Superficial site infections and sepsis tended to be more common after discharge than before discharge.

Reoperation occurred in $5 \%$ of patients $(n=170)$. Reoperations were equally likely to occur during index hospitalization as after discharge (Figure 1B). The most frequently coded reoperation within 30 days was hematoma evacuation ( $n=33,1 \%$ of all patients), which occurred a median of 2 days post-operatively (IQR 0-6), followed by the placement of external ventricular drain (EVD) or cerebrospinal fluid (CSF) shunt $(n=22,0.6 \%)$, which occurred after a median of 3 days (range 0-6) and 13 days (IQR 6-16) respectively. Among hematoma evacuations, $28 \%$ occurred on the same day as the index procedure while another $21 \%$ occurred on the first postoperative day.

Unplanned readmission was observed in $12 \%$ of cases $(\mathrm{n}=427)$. The most frequent indications surgical site infections (SSI) $(n=39,1 \%)$, non-infectious cardiac or pulmonary diseases $(\mathrm{n}=28,0.8 \%)$, oncological management of metastasis $(\mathrm{n}=28,0.8 \%)$, and pneumonia $(\mathrm{n}=27,0.8 \%)$.

Death within 30 days of a craniotomy occurred in $4 \%$ of patients and demonstrated wide temporal trends related to discharge. While the majority of 30-day deaths occurred after discharge $(72 \%)$, over a quarter were within the index hospitalization. Amongst patients with tracked mortality in this cohort, $66 \%$ were male and the median age was 63 years (IQR: 55 - 71). These patients were sicker than those who did not die: $32 \%$ of patients who died within 30 days were frail compared to $16 \%$ of those who did not. Furthermore, death after discharge occurred in $7 \%$ of patients requiring a reoperation compared to $3 \%$ of patients who did not (OR 2.4, p = 0.006). Similarly, death after discharge occurred in $12 \%$ of readmitted patients compared with $2 \%$ of non-readmitted patients (OR 7.7, p $<0.001$ ). 
TABLE 2 | Prevalence of complications stratified by index hospitalization.

\begin{tabular}{|c|c|c|c|c|c|c|c|}
\hline & \multirow{2}{*}{$\frac{\text { Total }}{\%}$} & \multicolumn{3}{|c|}{ Pre-Discharge } & \multicolumn{3}{|c|}{ Post-Discharge } \\
\hline & & $\%$ & Median (days) & IQR & $\%$ & Median (days) & IQR \\
\hline Superficial Site Infection & 1.49 & 0.26 & 13 & $10-14$ & 1.23 & 14 & $12-21.52$ \\
\hline Wound Dehiscence & 0.14 & 0.03 & 1 & $1-1$ & 0.11 & 20 & $16.5-23.8$ \\
\hline Pneumonia & 2.74 & 1.51 & 6 & $2-9$ & 1.14 & 18 & $15-23.5$ \\
\hline Reintubation & 1.74 & 1.03 & 3 & $2-7.8$ & 0.71 & 14 & $7-19.25$ \\
\hline Urinary Tract Infection & 2.14 & 1.00 & 5 & $0.5-6$ & 1.09 & 13.5 & $9.25-21.5$ \\
\hline Sepsis/Septic Shock & 2.09 & 0.66 & 6 & $5-9.5$ & 1.43 & 17 & $14-26$ \\
\hline VTE & 3.11 & 1.20 & 4.5 & $2-7.8$ & 1.91 & 17 & $14-22.5$ \\
\hline Cerebrovascular Accident & 1.06 & 0.60 & 2 & $1-6$ & 0.46 & 14 & $10-18.5$ \\
\hline Cardiac Arrest/Ml & 0.43 & 0.17 & 0 & $0-0$ & 0.26 & 20 & $18-24$ \\
\hline Reoperation & 4.86 & 2.49 & 3 & $2-7.5$ & 2.37 & 16 & $12-23.5$ \\
\hline Unplanned Readmission & 12.20 & & - & - & 12.20 & 17 & $11-23$ \\
\hline Death & 4.26 & 1.17 & 10 & $6-15$ & 3.09 & 20 & $15-26$ \\
\hline
\end{tabular}

IQR, Interquartile range; MI, Myocardial infarction; VTE, venous thromboembolism.

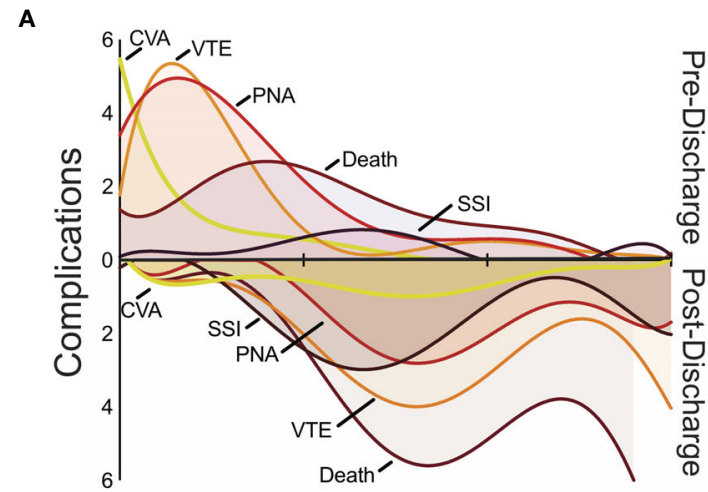

B
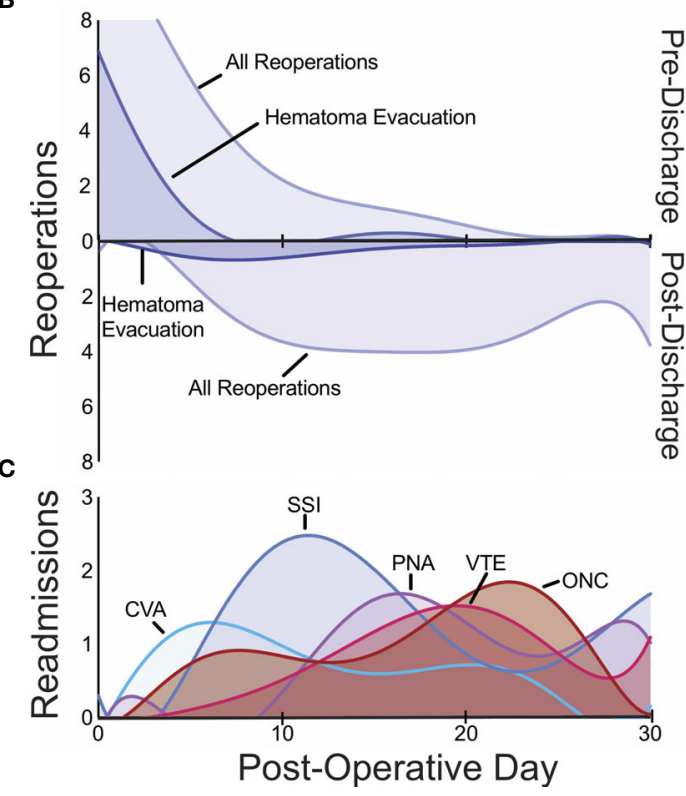

FIGURE 1 | Temporal patterns in 30-day pre- and post-discharge medical complications (A), pre- and post-discharge reoperations (B), and readmissions $\mathbf{( C )}$ with the day of the index operation defined as postoperative day 0. CSF, cerebrospinal fluid; CVA, cerebrovascular accident; ONC, medical oncologic admission; PNA, pneumonia; SSI, surgical site infection; VTE venous thromboembolism.

\section{Impact of Tumor Location}

We hypothesized that tumor location might affect the risk for a post-operative complication and mortality. Patients with infratentorial metastasis demonstrated similar demographic and co-morbid characteristics as those with supratentorial metastases, including age, gender, BMI, prior smoking history, CHF, COPD, hypertension, and diabetes. Craniotomies for infratentorial lesions were associated with an average operative time 33 minutes longer than supratentorial approaches $(\mathrm{p}<0.001)$.

Infratentorial location was found to be associated with increased odds for multiple 30-day medical complications, reoperation, and unplanned readmission (all p < 0.05) (Table 3). In particular, it was associated with increased odds of SSI (OR 2.9), pneumonia (OR 1.6), and reintubation (OR 1.9). It was also associated with length of stay above 7 days (OR 1.3) (Figure 2). Interestingly, infratentorial location was associated with decreased odds of VTE (OR 0.6).

Focusing on those cases with reoperation, infratentorial location was associated specifically with reoperations during the index hospitalization but not after discharge (Table 3). Amongst infratentorial lesions, $27 \%$ of reoperations were for CSF shunting (not including lumbar drains), 15\% entailed evacuation of a hematoma, and $11 \%$ were for placement of EVD. Placement of EVD or CSF shunt after the initial craniotomy for tumor was significantly more likely for infratentorial metastases than supratentorial metastases (OR 7.1).

Concordantly, infratentorial location was associated with significant risk of unplanned readmission for hydrocephalus (OR 5.1) (Table 3). The presence of hydrocephalus correlated with reoperation for CSF shunting or EVD placement for infratentorial lesions: $75 \%$ of hydrocephalus cases received either CSF shunt or EVD compared to $0.4 \%$ of cases without hydrocephalus $\left(\chi^{2}, \mathrm{p}<\right.$ 0.001 ). Readmissions for electrolyte/metabolic derangements (OR 4.5 ) and surgical site infections (OR 2.5) were also more common following infratentorial approaches. There was no statistically significant association between tumor location and death $(\mathrm{p}=0.77)$.

\section{Impact of Frailty}

We further assessed the association of frailty, as a composite reflection of chronic disease burden, and postoperative 
TABLE 3 | Adjusted association of infratentorial approach with 30-day postoperative complications.

\begin{tabular}{|c|c|c|c|c|c|c|}
\hline Medical Complications & $\begin{array}{c}\text { Total } \\
\%\end{array}$ & $\begin{array}{c}\text { Infratentorial } \\
\%\end{array}$ & $\begin{array}{c}\text { Supratentorial } \\
\%\end{array}$ & OR & $95 \% \mathrm{Cl}$ & $\mathbf{p}$ \\
\hline Surgical Site Infection & 1.49 & 2.90 & 1.05 & 2.92 & $1.62-5.28$ & $<0.001$ \\
\hline Wound Dehiscence & 0.14 & 0.24 & 0.11 & 1.95 & $\begin{array}{l}0.15- \\
25.76\end{array}$ & 0.613 \\
\hline Pneumonia & 2.74 & 3.99 & 2.36 & 1.63 & $1.01-2.63$ & 0.047 \\
\hline Reintubation & 1.74 & 3.02 & 1.34 & 1.90 & $1.08-3.33$ & 0.026 \\
\hline Venous & 3.11 & 2.06 & 3.44 & 0.55 & $0.32-0.95$ & 0.032 \\
\hline \multicolumn{7}{|l|}{ Thromboembolism } \\
\hline Urinary Tract Infection & 2.14 & 1.93 & 2.13 & 0.83 & $0.46-1.49$ & 0.529 \\
\hline Cerebrovascular Accident & 1.06 & 1.09 & 1.05 & 0.81 & $0.36-1.79$ & 0.597 \\
\hline Cardiac Arrest/Ml & 0.43 & 0.36 & 0.45 & 0.67 & $0.17-2.58$ & 0.559 \\
\hline Transfusion & 3.11 & 2.18 & 3.33 & 0.47 & $0.27-0.83$ & 0.009 \\
\hline Sepsis/Septic Shock & 2.09 & 2.42 & 1.98 & 1.19 & $0.68-2.09$ & 0.534 \\
\hline Extended Length of Stay* & 14.34 & 18.50 & 13.08 & 1.32 & $1.05-1.66$ & 0.018 \\
\hline Reoperation & 4.86 & 6.65 & 4.30 & 1.57 & $1.10-2.23$ & 0.013 \\
\hline Pre-Discharge ${ }^{\star \star}$ & 2.37 & 3.87 & 1.91 & 2.03 & $1.25-3.32$ & 0.004 \\
\hline Post-Discharge & 2.52 & 2.82 & 2.42 & 1.17 & $0.70-1.94$ & 0.552 \\
\hline Hematoma Evacuation & 0.94 & 0.97 & 0.93 & 1.24 & $0.52-2.96$ & 0.633 \\
\hline EVD/CSF Shunt & 0.63 & 2.06 & 0.37 & 7.11 & $2.88-$ & $<0.001$ \\
\hline Placement & & & & & 17.56 & \\
\hline Tumor Resection & 0.57 & 0.73 & 0.52 & 1.86 & $0.65-5.30$ & 0.247 \\
\hline Abscess Drainage & 0.46 & 0.36 & 0.49 & 0.93 & $0.24-3.64$ & 0.918 \\
\hline Unplanned Readmissions ${ }^{\star *}$ & 12.32 & 15.46 & 11.35 & 1.45 & $1.14-1.84$ & 0.002 \\
\hline Surgical Site Infection & 1.10 & 1.84 & 0.87 & 2.54 & $1.25-5.15$ & 0.010 \\
\hline Cardiac/Pulm Disease & 0.81 & 1.35 & 0.64 & 2.30 & $0.99-5.33$ & 0.053 \\
\hline Brain Metastases & 0.81 & 0.86 & 0.79 & 1.31 & $0.53-3.23$ & 0.560 \\
\hline Pneumonia & 0.78 & 0.61 & 0.83 & 0.90 & $0.32-2.54$ & 0.837 \\
\hline Seizure & 0.75 & 0.37 & 0.87 & 0.36 & $0.10-1.28$ & 0.115 \\
\hline Primary Cancer & 0.69 & 0.61 & 0.72 & 0.62 & $0.20-1.91$ & 0.408 \\
\hline $\mathrm{Gl}$ & 0.72 & 0.98 & 0.64 & 1.72 & $0.70-4.21$ & 0.237 \\
\hline VTE & 0.66 & 0.86 & 0.61 & 1.38 & $0.51-3.74$ & 0.522 \\
\hline CVA & 0.58 & 0.49 & 0.61 & 0.82 & $0.24-2.76$ & 0.745 \\
\hline Electrolyte/Metabolic & 0.49 & 0.98 & 0.34 & 4.46 & $\begin{array}{l}1.52- \\
13.07\end{array}$ & 0.007 \\
\hline Sepsis/Septic Shock & 0.38 & 0.12 & 0.45 & 0.27 & $0.03-2.23$ & 0.225 \\
\hline Hydrocephalus & 0.35 & 0.86 & 0.19 & 5.11 & $\begin{array}{l}1.32- \\
19.68\end{array}$ & 0.018 \\
\hline Death & 4.26 & 4.11 & 4.30 & 0.94 & $0.61-1.43$ & 0.765 \\
\hline Post-Discharge ${ }^{\star \star}$ & 3.12 & 2.70 & 3.25 & 0.83 & $0.50-1.37$ & 0.462 \\
\hline Pre-Discharge & 1.17 & 1.45 & 1.08 & 1.37 & $0.65-2.90$ & 0.405 \\
\hline
\end{tabular}

*excluding include 4 missing data points.

${ }^{* *}$ excluding 41 cases that died on index hospitalization.

Cl, Confidence interval; CSF, Cerebrospinal fluid; CVA, Cerebrovascular Accident; EVD, External ventricular drain; Gl, Gastrointestinal; MI, Myocardial Infarction; OR, Odds ratio; Pulm,

Pulmonary; VTE, venous thromboembolism.

outcomes. Frail patients had significantly higher likelihood of developing pneumonia (OR 2.8) (Table 4). Frailty was also associated with increased risk of reoperation on the index hospitalization compared to non-frail patients (OR 2.0), but not reoperation after discharge (Figure 2). Frail patients were especially at risk for surgical evacuation of hematoma (OR 3.6). Frailty was not associated with unplanned readmission overall or any specific reasons for readmission. Pre-frailty and frailty had elevated odds ratios of 1.8 and 2.6 for death compared to nonfrail patients, respectively. On temporal analysis, frailty was associated with death after discharge, but not before discharge.

\section{Predictive Model for Pre-Mature Mortality}

A multivariate logistic regression analysis with stepwise variable elimination was performed to construct a predictive model for death within 30 days after surgery for brain metastasis (Table 5).
Notably, frailty and pre-frailty were significant predictors in this model. Thrombocytopenia below 100,000 cells/dL had the highest strength of association of any variable. Additional predictors within the model included ASA score of $4 / 5$, male sex, age over 75 years, other preoperative laboratory abnormalities, non-elective case designation, and preoperative steroid use. The model demonstrated a fair ability to correctly classify patients who die prematurely (AUROC 0.75).

\section{DISCUSSION}

Determining which patients will benefit from surgical resection of brain metastases requires balancing potential improvements in overall survival and local control with the risks of surgery, hospitalization, and rehabilitation. We provide a global 


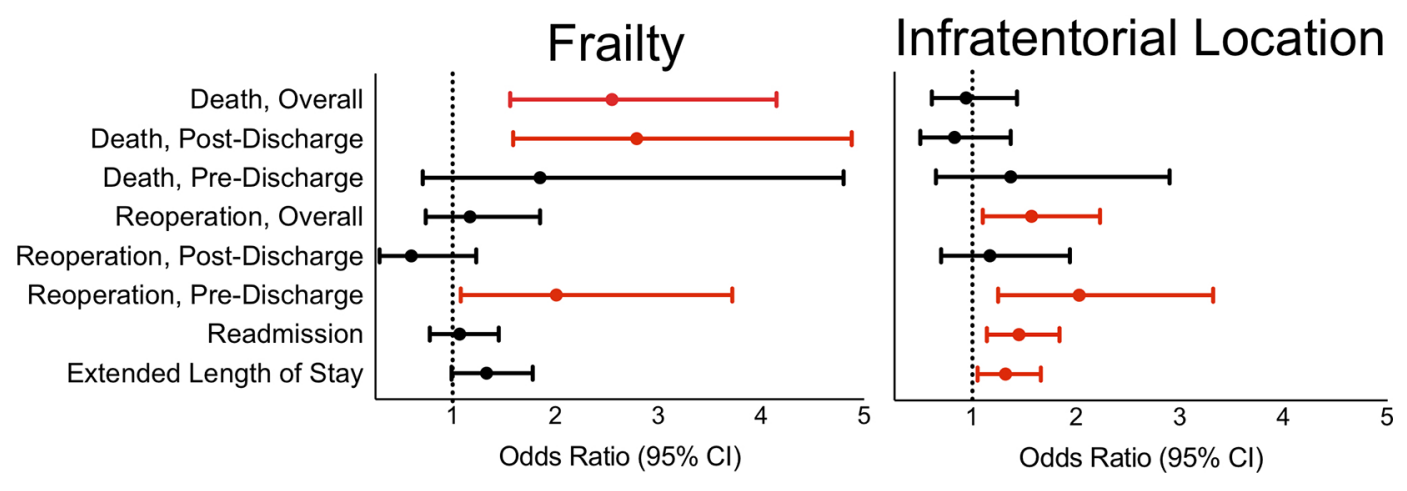

FIGURE 2 | In reference to supratentorial approaches, infratentorial surgical approach is associated with length of stay over 7 days, 30 -day overall and predischarge reoperation, and unplanned readmission. In reference to non-frailty, frailty is associated with 30 -day pre-discharge reoperation as well as overall and postdischarge death.

TABLE 4 | Adjusted association of pre-frailty and frailty with 30-day postoperative complications compared with non-frail patients.

\begin{tabular}{|c|c|c|c|c|c|c|c|c|c|}
\hline \multirow[b]{2}{*}{ Medical Complications } & \multirow{2}{*}{$\frac{\text { Non-Frail }}{\%}$} & \multicolumn{4}{|c|}{ Pre-Frail } & \multicolumn{4}{|c|}{ Frail } \\
\hline & & $\%$ & OR & $95 \% \mathrm{Cl}$ & $\mathbf{p}$ & $\%$ & OR & $95 \% \mathrm{Cl}$ & $\mathbf{p}$ \\
\hline Surgical Site Infection & 1.91 & 0.88 & 0.43 & $0.21-0.90$ & 0.026 & 1.54 & 0.80 & 0.34-1.86 & 0.600 \\
\hline Dehiscence & 0.18 & 0.08 & 0.22 & $0.02-2.95$ & 0.250 & 0.17 & 0.35 & $0.02-5.45$ & 0.453 \\
\hline Pneumonia & 1.91 & 2.17 & 1.02 & $0.59-1.79$ & 0.933 & 6.35 & 2.78 & $1.59-4.87$ & $<0.001$ \\
\hline Reintubation & 1.26 & 1.69 & 1.05 & $0.55-2.02$ & 0.875 & 3.26 & 1.64 & 0.80-3.36 & 0.175 \\
\hline VTE & 2.99 & 2.73 & 0.78 & $0.48-1.26$ & 0.315 & 4.29 & 1.16 & $0.67-2.03$ & 0.595 \\
\hline Urinary Tract Infection & 1.38 & 2.49 & 1.52 & $0.84-2.73$ & 0.167 & 3.26 & 1.56 & $0.78-3.14$ & 0.212 \\
\hline Cerebrovascular Accident & 0.66 & 1.53 & 1.84 & $0.82-4.14$ & 0.141 & 1.20 & 1.13 & $0.39-3.26$ & 0.827 \\
\hline Cardiac Arrest/Ml & 0.30 & 0.32 & 0.57 & $0.14-2.31$ & 0.431 & 1.03 & 1.40 & $0.38-5.17$ & 0.617 \\
\hline Transfusion & 2.39 & 3.13 & 1.18 & $0.70-1.98$ & 0.537 & 4.81 & 1.27 & $0.69-2.34$ & 0.435 \\
\hline Sepsis/Septic Shock & 1.44 & 2.17 & 1.18 & $0.65-2.14$ & 0.587 & 3.77 & 1.64 & $0.84-3.20$ & 0.145 \\
\hline Extended Length of Stay* & 12.03 & 14.88 & 1.14 & $0.90-1.45$ & 0.283 & 19.93 & 1.33 & $0.99-1.78$ & 0.056 \\
\hline Reoperation & 4.67 & 4.58 & 0.91 & $0.62-1.33$ & 0.631 & 6.00 & 1.17 & $0.74-1.85$ & 0.510 \\
\hline Pre-Discharge & 1.91 & 2.17 & 1.13 & $0.64-1.97$ & 0.679 & 4.12 & 2.01 & 1.08-3.72 & 0.027 \\
\hline Post-Discharge ${ }^{\star \star}$ & 2.77 & 2.44 & 0.79 & $0.48-1.30$ & 0.352 & 1.93 & 0.60 & $0.29-1.23$ & 0.159 \\
\hline Hematoma Evacuation & 0.54 & 1.20 & 2.63 & $1.04-6.60$ & 0.040 & 1.54 & 3.58 & $1.24-10.32$ & 0.018 \\
\hline EVD/CSF Shunt Placement & 0.60 & 0.40 & 0.63 & $0.22-1.80$ & 0.384 & 1.20 & 1.57 & $0.53-4.64$ & 0.410 \\
\hline Tumor Resection & 0.90 & 0.32 & 0.34 & $0.10-1.12$ & 0.075 & 0.17 & 0.17 & $0.02-1.46$ & 0.106 \\
\hline Abscess Drainage & 0.66 & 0.24 & 0.30 & $0.07-1.24$ & 0.097 & 0.34 & 0.58 & $0.10-3.24$ & 0.537 \\
\hline Unplanned Readmission & 11.51 & 12.46 & 0.97 & $0.76-1.24$ & 0.823 & 14.36 & 1.07 & $0.78-1.45$ & 0.686 \\
\hline Surgical Site Infection & 1.39 & 0.73 & 0.45 & $0.19-1.06$ & 0.067 & 1.05 & 0.75 & $0.27-2.09$ & 0.581 \\
\hline Cardiac/Pulm Disease & 0.54 & 0.73 & 0.94 & $0.34-2.62$ & 0.913 & 1.75 & 2.37 & $0.84-6.72$ & 0.104 \\
\hline Brain Metastases & 0.78 & 0.98 & 1.29 & $0.56-3.00$ & 0.553 & 0.53 & 0.73 & $0.19-2.83$ & 0.647 \\
\hline Pneumonia & 0.54 & 0.90 & 1.82 & $0.69-4.80$ & 0.225 & 1.23 & 2.41 & $0.78-7.44$ & 0.127 \\
\hline Seizure & 0.66 & 0.73 & 0.81 & $0.31-2.13$ & 0.665 & 1.05 & 1.24 & $0.39-3.96$ & 0.713 \\
\hline Primary Cancer & 0.66 & 0.98 & 1.37 & $0.53-3.53$ & 0.513 & 0.18 & 0.17 & $0.02-1.53$ & 0.113 \\
\hline $\mathrm{Gl}$ & 0.66 & 0.81 & 1.04 & $0.41-2.63$ & 0.932 & 0.70 & 0.71 & $0.20-2.52$ & 0.591 \\
\hline VTE & 0.60 & 0.73 & 0.90 & $0.34-2.41$ & 0.832 & 0.70 & 0.84 & 0.24-3.01 & 0.792 \\
\hline CVA & 0.30 & 0.98 & 2.36 & $0.76-7.32$ & 0.138 & 0.53 & 1.15 & $0.24-5.54$ & 0.865 \\
\hline Electrolyte/Metabolic & 0.54 & 0.41 & 0.70 & $0.21-2.29$ & 0.554 & 0.53 & 0.90 & 0.21-3.82 & 0.892 \\
\hline Sepsis/Septic Shock & 0.18 & 0.49 & 2.26 & $0.5-10.24$ & 0.289 & 0.70 & 2.91 & $0.52-16.19$ & 0.222 \\
\hline Hydrocephalus & 0.30 & 0.41 & 0.93 & $0.23-3.69$ & 0.916 & 0.35 & 0.40 & $0.04-3.67$ & 0.419 \\
\hline Death & 2.45 & 4.82 & 1.76 & $1.13-2.74$ & 0.012 & 8.23 & 2.55 & $1.56-4.15$ & $<0.001$ \\
\hline Post-Discharge & 1.75 & 3.50 & 1.72 & $1.04-2.87$ & 0.036 & 6.30 & 2.79 & $1.59-4.88$ & $<0.001$ \\
\hline Pre-Discharge & 0.72 & 1.37 & 1.75 & $0.74-4.09$ & 0.200 & 2.06 & 1.85 & $0.71-4.80$ & 0.208 \\
\hline
\end{tabular}

*4 cases with missing length of stay not included.

**excluding 41 cases that died on index hospitalization.

Cl, Confidence interval; CSF, Cerebrospinal fluid; CVA, Cerebrovascular Accident; EVD, External ventricular drain; Gl, Gastrointestinal; MI, Myocardial Infarction; OR, Odds ratio; Pulm, Pulmonary; VTE, venous thromboembolism. 
TABLE 5 | Multivariate logistic regression predictive model for 30-day death sorted by predictor effect size.

\begin{tabular}{|c|c|c|c|}
\hline Predictor & OR & $95 \% \mathrm{Cl}$ & p-value \\
\hline Thrombocytopenia (under 100,000 cells/dL) & 3.41 & $1.32-7.69$ & 0.006 \\
\hline ASA score of $4-5$ & 3.05 & $1.38-8.09$ & 0.012 \\
\hline Frailty & 2.48 & $1.59-3.89$ & $<0.001$ \\
\hline Male Sex & 2.18 & $1.53-3.14$ & $<0.001$ \\
\hline Age $75+$ & 2.00 & $1.24-3.12$ & 0.003 \\
\hline WBCs above 12,000 cells/dL & 1.88 & $1.33-2.66$ & $<0.001$ \\
\hline ASA score of 3 & 1.79 & $0.83-4.66$ & 0.179 \\
\hline Anemia (hematocrit 24-36\%) & 1.73 & $1.20-2.46$ & 0.003 \\
\hline Urgent/Emergent Case & 1.68 & $1.17-2.43$ & 0.005 \\
\hline Pre-frailty & 1.62 & $1.09-2.50$ & 0.021 \\
\hline Pre-operative Steroid Use & 1.58 & $1.08-2.29$ & 0.017 \\
\hline Hyponatremia & 1.40 & $0.93-2.08$ & 0.098 \\
\hline AUROC & & & 0.754 \\
\hline Hosmer-Lemeshow Statistic & & & 0.211 \\
\hline
\end{tabular}

ASA, American Society of Anesthesiologists; Cl, Confidence interval; OR, Odds ratio; WBC, White blood cells.

Predictors that did not meet criteria for statistical significance but that remained in the model after stepwise variable elimination are italicized.

perspective for this common diagnosis and a predictive tool for 30-day mortality risk.

Post-operative complications during the index hospitalization demonstrated clear temporal trends and did not fit neatly into the classic "wind, water, wound, walk" surgical teaching paradigm (23). This cohort experienced early, but infrequent cardiac events usually the day of operation; cerebrovascular accidents at a median 2 days post-operatively; UTIs and pneumonia at a median of 5-6 days post-operatively; and surgical site infections 2 weeks after operation, suggesting that SSIs may be more commonly related to post-operative seeding from sources such as bedding and dressing changes. Awareness of the peak incidence for these complications may highlight tailored screening and follow-up when clinically indicated. Infratentorial approaches for tumor resection were associated with higher rates of pneumonia and reintubation, which are pulmonary complications potentially attributed to aspiration events and dysphagia related to cranial nerve dysfunction (24-26).

Reoperations occurred in a minority of patients, with the most common reason for reoperation being evacuation of intracranial hematoma. This usually occurred in the perioperative period. We found that craniotomies for infratentorial pathologies were associated with reoperation for CSF diversion, with similar rates of this potential complication during the index hospitalization or after discharge, suggesting the need for vigilance against hydrocephalus in both early and delayed settings. Frailty was associated with higher risk of reoperation before discharge but not post-discharge, as well as risk for hematoma evacuation.

More so than for other neurosurgical patients, for those with guarded prognoses, attempts to maximize quality of life and time spent with family or out of the hospital become tantamount. Surgery for infratentorial lesions had increased odds of readmission overall and for readmission associated with hydrocephalus, electrolyte/metabolic derangements, and pneumonia. The association with hydrocephalus is consistent with the elevated odds of intracranial pressure-reducing surgery observed for infratentorial metastases. Further, the proximity of the third ventricle to the hypothalamus may explain the relationship between hydrocephalus and electrolyte anomalies previously observed. The reasons for the lack of association between frailty and readmission may be multifactorial, including bias in the cohort studied or the goals of care for brain metastases patients, for whom palliation may be considered over hospitalization when non-operative complications arise.

The incidence of 30-day death for brain metastases was similar to that reported in other series, and slightly higher than rates observed for other intracranial tumors (27-30). This rate is improved compared to in-hospital postoperative mortality of 3\% for brain metastases observed from 1988-2000, which may reflect improved operative management, perioperative care, and patient selection (31). Death occurred more frequently after discharge, though only $1 \%$ of all patients died prior to discharge. Given the importance of assessing postoperative mortality risk, we developed a predictive model for 30-day mortality that revealed high ASA score, frailty, male sex, thrombocytopenia amongst other preoperative laboratory abnormalities, case urgency, and preoperative steroid usage to predict mortality. Certain factors included in this model, like frailty and ASA score, are proxies for well-being as well as chronic disease burden and increased nutritional and medical needs that are best met in the inpatient setting, leading to a deterioration after discharge. This tool may augment surgical experience for borderline cases. Importantly, factors that are included may not have a mechanistic link to the outcome but may be proxy metrics for correlates that were not assessed. Factors that are not included in a model may still be important to consider in the management of the patient, but do not provide an added benefit in the predictive capacity of an empiric model.

Providers managing patients after surgery should be cognizant of the expected time course for common perioperative complications and adjust surveillance and followup. While tumor location may not be a modifiable factor, monitoring after surgery is. Implementing protocols to avoid pulmonary complications and close monitoring for signs of increased intracranial pressure may mitigate the risks 
associated with the infratentorial location for example (32). Optimization of elements that contribute to frailty, including blood pressure and blood glucose, could further decrease the additional per-operative risks incurred by patients. Given the association between frailty and post-discharge death in particular, frail patients would benefit from an assessment of their understanding regarding postoperative and chronic medical needs, medications, and appointments. Involving patients' family members and caregivers in their medical planning may assist in these efforts.

This study has pertinent limitations in methodology. We capture 3,500 cases, which is likely a small proportion of all brain metastasis resections within this time frame (2). The NSQIP database only encapsulates complications up to 30 days after operation, which is generally below the expected survival of brain metastasis patients and thus limits long-term predictions. Supratentorial tumors have a wide range of operative risk based on eloquence, language dominance and lobe, which is not captured in this study, and are important for further study. For example, a complication for a metastasis near the motor cortex may cause hemiplegia and significantly decrease quality of life, which would not be captured in these data. Hospitals are not required to report all surgeries that occur at their institution, thus selection bias can be introduced depending on those cases the hospital chooses to report. There may also be confounding by hospital type, size, and specialization as hospital-level data are not available. The NSQIP database does not contain oncologic parameters relevant to the care of brain metastasis patients, including size and number of metastases, symptomatology, neo-adjuvant chemo- or radiotherapy, and histology, and does not track patients' quality of life and overall survival. There are no data included on post-operative thromboprophylaxis.

\section{CONCLUSION}

Selecting candidates for brain metastasis resection deliberately balances anticipated benefits to quality of life and survival with post-operative risks and recovery time. Tumor location and patient frailty expose patients to differing post-operative risks

\section{REFERENCES}

1. Barnholtz-Sloan JS, Sloan AE, Davis FG, Vigneau FD, Lai P, Sawaya RE. Incidence Proportions of Brain Metastases in Patients Diagnosed (1973 to 2001) in the Metropolitan Detroit Cancer Surveillance System. J Clin Oncol (2004) 22(14):2865-72. doi: 10.1200/JCO.2004.12.149

2. Cagney DN, Martin AM, Catalano PJ, Redig AJ, Lin NU, Lee EQ, et al. Incidence and Prognosis of Patients With Brain Metastases At Diagnosis of Systemic Malignancy: A Population-Based Study. Neuro Oncol (2017) 19 (11):1511-21. doi: 10.1093/neuonc/nox077

3. Nuno M, Mukherjee D, Elramsisy A, Nosova K, Lad SP, Boakye M, et al. Racial and Gender Disparities and the Role of Primary Tumor Type on Inpatient Outcomes Following Craniotomy for Brain Metastases. Ann Surg Oncol (2012) 19(8):2657-63. doi: 10.1245/s10434-012-2353-z

4. Nayak L, Lee EQ, Wen PY. Epidemiology of Brain Metastases. Curr Oncol Rep (2012) 14(1):48-54. doi: 10.1007/s11912-011-0203-y and should be considered in personalized surgical decisionmaking. We highlight our predictive tool for 30-day mortality in these patients that incorporates frailty as well as other demographic and clinical factors.

\section{DATA AVAILABILITY STATEMENT}

The datasets presented in this study can be found in online repositories. The names of the repository/repositories and accession number(s) can be found below: https://www.facs.org/ Quality-Programs/ACS-NSQIP.

\section{ETHICS STATEMENT}

Ethical review and approval was not required for the study on human participants in accordance with the local legislation and institutional requirements. Written informed consent for participation was not required for this study in accordance with the national legislation and the institutional requirements.

\section{AUTHOR CONTRIBUTIONS}

The conceptualization and design of this work was performed by SG, HD, LF, EH, ID, and WB. Data extraction and preparation was performed by SG, AGL, HD, and LF. Data analysis was performed by SG, AG, HD, LF, and EH. Data interpretation was performed by all authors. Manuscript drafting was performed by SG, AG, HD, LF, EH, EL, AA, and WB. All authors contributed to the article and approved the submitted version.

\section{SUPPLEMENTARY MATERIAL}

The Supplementary Material for this article can be found online at: https://www.frontiersin.org/articles/10.3389/fonc.2021. 662943/full\#supplementary-material
5. Smedby KE, Brandt L, Backlund ML, Blomqvist P. Brain Metastases Admissions in Sweden Between 1987 and 2006. Br J Cancer (2009) 101 (11):1919-24. doi: 10.1038/sj.bjc.6605373

6. Lee SS, Ahn JH, Kim MK, Sym SJ, Gong G, Ahn SD, et al. Brain Metastases in Breast Cancer: Prognostic Factors and Management. Breast Cancer Res Treat (2008) 111(3):523-30. doi: 10.1007/s10549-007-9806-2

7. Choong ES, Lo S, Drummond M, Fogarty GB, Menzies AM, Guminski A, et al. Survival of Patients With Melanoma Brain Metastasis Treated With Stereotactic Radiosurgery and Active Systemic Drug Therapies. Eur J Cancer (2017) 75:169-78. doi: 10.1016/j.ejca.2017.01.007

8. Ali A, Goffin JR, Arnold A, Ellis PM. Survival of Patients With non-Small-Cell Lung Cancer After a Diagnosis of Brain Metastases. Curr Oncol (2013) 20(4): e300-6. doi: 10.3747/co.20.1481

9. Sperduto PW, Yang TJ, Beal K, Pan H, Brown PD, Bangdiwala A, et al. Estimating Survival in Patients With Lung Cancer and Brain Metastases: an Update of the Graded Prognostic Assessment for Lung Cancer Using 
Molecular Markers (Lung-MolGPA). JAMA Oncol (2017) 3(6):827-31. doi: 10.1001/jamaoncol.2016.3834

10. Owonikoko TK, Arbiser J, Zelnak A, Shu H-KG, Shim H, Robin AM, et al. Current Approaches to the Treatment of Metastatic Brain Tumours. Nat Rev Clin Oncol (2014) 11(4):203-22. doi: 10.1038/nrclinonc.2014.25

11. Reyes DK, Pienta KJ. The Biology and Treatment of Oligometastatic Cancer. Oncotarget (2015) 6(11):8491-524. doi: 10.18632/oncotarget. 3455

12. Patchell RA. The Management of Brain Metastases. Cancer Treat Rev (2003) 29(6):533-40. doi: 10.1016/S0305-7372(03)00105-1

13. Patchell RA, Cirrincione C, Thaler HT, Galicich JH, Kim JH, Posner JB. Single Brain Metastases: Surgery Plus Radiation or Radiation Alone. Neurology (1986) 36(4):447-53. doi: 10.1212/WNL.36.4.447

14. Schodel P, Schebesch KM, Brawanski A, Proescholdt MA. Surgical Resection of Brain Metastases-Impact on Neurological Outcome. Int J Mol Sci (2013) 14 (5):8708-18. doi: 10.3390/ijms14058708

15. Vecht CJ, Haaxma-Reiche H, Noordijk EM, Padberg GW, Voormolen JH, Hoekstra FH, et al. Treatment of Single Brain Metastasis: Radiotherapy Alone or Combined With Neurosurgery? Ann Neurol (1993) 33(6):583-90. doi: 10.1002/ana.410330605

16. Chimukangara M, Helm MC, Frelich MJ, Bosler ME, Rein LE, Szabo A, et al. A 5-Item Frailty Index Based on NSQIP Data Correlates With Outcomes Following Paraesophageal Hernia Repair. Surg Endosc (2017) 31(6):2509-19. doi: 10.1007/s00464-016-5253-7

17. Dasgupta M, Rolfson DB, Stolee P, Borrie MJ, Speechley M. Frailty is Associated With Postoperative Complications in Older Adults With Medical Problems. Arch Gerontol Geriatr (2009) 48(1):78-83. doi: 10.1016/j.archger.2007.10.007

18. Fried LP, Tangen CM, Walston J, Newman AB, Hirsch C, Gottdiener J, et al. Frailty in Older Adults: Evidence for a Phenotype. J Gerontol A Biol Sci Med Sci (2001) 56(3):M146-56. doi: 10.1093/gerona/56.3.M146

19. Lee DH, Buth KJ, Martin BJ, Yip AM, Hirsch GM. Frail Patients are At Increased Risk for Mortality and Prolonged Institutional Care After Cardiac Surgery. Circulation (2010) 121(8):973-8. doi: 10.1161/CIRCULATIONAHA.108.841437

20. Woods NF, LaCroix AZ, Gray SL, Aragaki A, Cochrane BB, Brunner RL, et al. Frailty: Emergence and Consequences in Women Aged 65 and Older in the Women's Health Initiative Observational Study. J Am Geriatr Soc (2005) 53 (8):1321-30. doi: 10.1111/j.1532-5415.2005.53405.x

21. Sellers MM, Merkow RP, Halverson A, Hinami K, Kelz RR, Bentrem DJ, et al. Validation of New Readmission Data in the American College of Surgeons National Surgical Quality Improvement Program. J Am Coll Surg (2013) 216 (3):420-7. doi: 10.1016/j.jamcollsurg.2012.11.013

22. Subramaniam S, Aalberg JJ, Soriano RP, Divino CM. New 5-Factor Modified Frailty Index Using American College of Surgeons NSQIP Data. J Am Coll Surg (2018) 226(2):173-81 e8. doi: 10.1016/j.jamcollsurg.2017.11.005

23. Pile JC. Evaluating Postoperative Fever: a Focused Approach. Cleve Clin J Med (2006) 73 Suppl 1:S62-6. doi: 10.3949/ccjm.73.Suppl_1.S62
24. Cai YH, Wang HT, Zhou JX. Perioperative Predictors of Extubation Failure and the Effect on Clinical Outcome After Infratentorial Craniotomy. Med Sci Monit (2016) 22:2431-8. doi: 10.12659/MSM.899780

25. Flexman AM, Merriman B, Griesdale DE, Mayson K, Choi PT, Ryerson CJ. Infratentorial Neurosurgery is an Independent Risk Factor for Respiratory Failure and Death in Patients Undergoing Intracranial Tumor Resection. J Neurosurg Anesthesiol (2014) 26(3):198-204. doi: 10.1097/ANA. 0b013e3182a43ed8

26. Park DH, Chun MH, Lee SJ, Song YB. Comparison of Swallowing Functions Between Brain Tumor and Stroke Patients. Ann Rehabil Med (2013) 37 (5):633-41. doi: 10.5535/arm.2013.37.5.633

27. Dasenbrock HH, Devine CA, Liu KX, Gormley WB, Claus EB, Smith TR, et al. Thrombocytopenia and Craniotomy for Tumor: a National Surgical Quality Improvement Program Analysis. Cancer (2016) 122(11):1708-17. doi: $10.1002 / \mathrm{cncr} .29984$

28. Dickinson H, Carico C, Nuno M, Mukherjee D, Ortega A, Black KL, et al. Unplanned Readmissions and Survival Following Brain Tumor Surgery. J Neurosurg (2015) 122(1):61-8. doi: 10.3171/2014.8.JNS1498

29. Lassen B, Helseth E, Egge A, Due-Tonnessen BJ, Ronning P, Meling TR. Surgical Mortality and Selected Complications in 273 Consecutive Craniotomies for Intracranial Tumors in Pediatric Patients. Neurosurgery (2012) 70(4):936-43; discussion 43. doi: 10.1227/NEU.0b013e31823bcc61

30. Lassen B, Helseth E, Ronning P, Scheie D, Johannesen TB, Maehlen J, et al. Surgical Mortality At 30 Days and Complications Leading to Recraniotomy in 2630 Consecutive Craniotomies for Intracranial Tumors. Neurosurgery (2011) 68(5):1259-68; discussion 68-9. doi: 10.1227/NEU.0b013e31820c0441

31. Barker FG,2. Craniotomy for the Resection of Metastatic Brain Tumors in the U.S., 1988-2000: Decreasing Mortality and the Effect of Provider Caseload. Cancer (2004) 100(5):999-1007. doi: 10.1002/cncr.20058

32. Cassidy MR, Rosenkranz P, McCabe K, Rosen JE, McAneny D. I COUGH: Reducing Postoperative Pulmonary Complications With a Multidisciplinary Patient Care Program. JAMA Surg (2013) 148(8):740-5. doi: 10.1001/ jamasurg.2013.358

Conflict of Interest : The authors declare that the research was conducted in the absence of any commercial or financial relationships that could be construed as a potential conflict of interest.

Copyright (c) 2021 Gupta, Dawood, Giantini Larsen, Fandino, Knelson, Smith, Lee, Aizer, Dunn and Bi. This is an open-access article distributed under the terms of the Creative Commons Attribution License (CC BY). The use, distribution or reproduction in other forums is permitted, provided the original author(s) and the copyright owner(s) are credited and that the original publication in this journal is cited, in accordance with accepted academic practice. No use, distribution or reproduction is permitted which does not comply with these terms. 